\title{
Implementation of QAM Modulation Demodulation Based on
}

\author{
Simulink
}

\author{
Wanjian Jiang,Mingjie Zhao, Yaodong Tang \\ Computer Technology and Engineering College, Qinhuangdao University, Hebei, China
}

\begin{abstract}
This paper is about Simulink QAM modulation and demodulation implementation. Firstly, the basic principle of QAM modulation and demodulation system is introduced. Then, the basic operation of system modeling and simulation under simulation software Simulink is introduced. The emphasis is on modeling and simulation of 16QAM system modules, and 16QAM modulation and demodulation are realized. Finally, a 2DPSK system simulation is carried out to compare it with the 16QAM system. It is concluded that 16QAM is a relatively superior modulation and demodulation system.
\end{abstract}

Key words:QAM modulation and demodulation; Simulink; simulation

\section{Chapter 1 Introduction}

1.1 Introduction and application of QMA

In rapidly developing information age, with the rapid development of digital communication technology and computer technology and the integration of communication network and computer network, information science and technology has become a new and powerful driving force in the 21 st century for international society and world economic development. Modern digital communication technology has three basic ways of modulation: digital amplitude modulation, digital frequency modulation, digital phase modulation, but these three digital modulation methods are deficiencies, such as: low spectral efficiency, anti-multipath resistance, poor ability, slow attenuation of power spectrum, serious externality and so on. In order to improve these shortcomings, in recent decades people have been put forward some new digital modulation and demodulation technology to meet the requirements of various communication systems. The main research contents focus on reducing the signal bandwidth to improve the signal spectrum utilization; improve power utilization to enhance the anti-noise performance; to adapt to a variety of channels with the enhanced anti-multipath antifading ability.

Quadrature Amplitude Modulation (QAM) is a kind of modulation method with high spectrum utilization. It is widely used in the fields of medium and large capacity digital microwave communication system, high speed data transmission of cable television network and satellite communication system. QAM digital modulator as a DVB system front-end equipment, from the encoder, multiplexer, DVB gateway, video server and other equipment TS stream, the RS coding, convolutional coding and QAM digital modulation, the output of the RF signal can direct transmits cable television network, but also depending to the need to select the IF output. It is widely used in the field of digital cable television transmission and digital MMDS system with its flexible configuration and superior performance index. For example, in the constant guru channel, the QAM scheme has high spectral efficiency, so quadrature amplitude modulation is widely used in the field of satellite communication and high-speed data transmission of cable television networks.
Mathworks introduced a mathematical application software called as Matlab, has now developed, including communication systems and a number of toolbox, which became the current scientific research and engineering applications, it is now one of the most popular software package. Simulink is a visual simulation tool in Matlab that provides an integrated environment for dynamic system modeling, simulation and comprehensive analysis. In Simulink, there is no need for a lot of writing procedures, and only through a simple mouse operation and other commands to establish an intuitive system block diagram model, the user can freely change the model of the module parameters, and can immediately see the change parameters, so as to achieve a convenient, fast modeling and simulation purposes. Simulink includes a complex receiver, a source, a linear and non-linear component, and a modular library of connections. Users can also customize or create their own modules as needed. It can be used in linear systems, nonlinear systems, digital control and digital signal processing modeling and simulation. Simulink has been widely used in control theory and digital signal processing and communication system simulation and design. Simulink has been widely used in control theory and digital signal processing and communication system simulation and design, and has the advantages of wide adaptability, clear structure and process, compactness, close to reality, fast and efficient, flexible and convenient.

The actual communication system is a system with a very complex functional structure, and any changes made to the system can affect the performance and stability of the entire system. Simulink, as a toolkit for modeling, simulating and analyzing dynamic systems provided by Matlab, provides the source code needed for simulation, error correction coding, channel, modem, and all other library functions and modules. The Visible, regardless of any complex communication system, with Simulink on its simulation is a good choice.

\subsection{The main content of the paper}

This paper is based on Simulink QAM modulation and demodulation implementation. Firstly, the basic principle of QAM modulation and demodulation system is introduced, and then the basic knowledge of simulink library and the basic operation of system modeling and simulation are introduced. The key point is to 16QAM modulation and demodulation system as an example, according to its 
modulation and demodulation principle system block diagram, in Simulink environment to establish the model of each module, such as serial and parallel conversion module, $2 / 4$ level conversion module, set the parameters and simulation. The modulation and demodulation of the QAM system are realized, and the waveforms, modulating and demodulating waveforms of the modules are obtained, and the constellations and eye patterns of the signals before and after the noise are obtained. Under the condition of SNR, the 16QAM system bit error rate. Finally, after making a 2DPSK system design and simulation, it is compared with the 16QAM system, and the conclusion that 16QAM is a relatively superior modulation and demodulation system is obtained.

\section{Chapter 2 Modern Digital Modulation Technology}

QAM is the abbreviation of the Quadrature Amplitude Modulation, which is a quadrature amplitude modulation, which is a digital modulation scheme for amplitude modulation on two orthogonal carriers. The two carriers are usually 90 degrees $(\pi / 2)$ sine wave, so called quadrature carrier, this modulation method get the name from here.

\subsection{QAM modulation principle}

The so-called quadrature amplitude modulation is to use two independent baseband waveforms on two mutually orthogonal cofrequency carriers to suppress the carrier of the double sideband modulation. Using this modulated signal in the same bandwidth within the spectrum of orthogonal nature to achieve two parallel digital signal transmissions. signal is

The general expression of the quadrature amplitude modulation

$(2-1)$

Where is the baseband signal amplitude, is the width of a single baseband signal waveform. The above formula is transformed into an orthogonal representation:

(2-2)

Order

Then (2-2) becomes

$=(2-3)$

The amplitude and in the equation can be expressed as

Where, A is fixed amplitude, there is input data of to determine. vetermines the coordinates of the QAM signal in the signal space.

The QAM modem block diagram is shown in Figure 2-1:

Figure 2-1 Schematic diagram of QAM signal demodulation

In the figure, the input binary sequence is converted to the twoway parallel sequence of the serial / parallel conversion output rate by half, and then the baseband signal of L level is formed by 2 to $\mathrm{L}$ level conversion respectively. The L-level baseband signal is also subjected to a pre-modulation low-pass filter in order to suppress the out-of-band radiation of the modulated signal, and after the LPF, $\mathrm{X}(\mathrm{t})$ and $\mathrm{Y}(\mathrm{t})$ are formed, cross-carrier multiplication, and finally the two signals plus QAM signal.

\subsection{QAM demodulation principle}

MQAM signal can use orthogonal coherent demodulation method, the demodulator principle shown in Figure 2-2:

Figure 2-2 MQAM signal coherent demodulation schematic

The demodulator input signal is multiplied by two orthogonal carriers recovered locally and then output through the low-pass filter to output two multi-level baseband signals $\mathrm{X}(\mathrm{t})$ and $\mathrm{Y}(\mathrm{t})$. (L-1) decision thresholds, where $\mathrm{L}$ is the number of levels, and then by $\mathrm{L}$ level to 2 level conversion and parallel / serial converter (multi-level decision device) on the multi-level baseband signal decision and detect the final output binary data.

\subsection{MQAM signal constellation}

The distribution of the endpoints of the signal vector is called the constellation. The spatial distribution of the QAM signal is usually described by a constellation. For 16QAM, there are a variety of distributed forms of signal constellation. As shown in Figure 2-3. In Figure 2-3 (a) the distribution of signal points into a square, so called square 16QAM constellation, also known as the standard 16QAM constellation. In Figure 2-3 (b) the distribution of signal points into stars, it is called the star 16QAM constellation.

Figure 2-3 Block diagram of 16QAM

(A) Square 16QAM constellation (b) star 16QAM constellation

$\mathrm{M}=4,16,32, \ldots, 256 \mathrm{MQAM}$ signal constellation Figure 2-4 shows:

Figure 2-4 MQAM signal constellation

Where $M$ is an even order of 2, such as $M=4,16,64,256$ when the constellation is rectangular, each symbol carries even bits of information; and $\mathrm{M}$ is 2 odd times, such as $\mathrm{M}=32,128$ constellation Cross, each symbol carries odd bits of information.

In order to compare the anti-jamming capability of MQAM and MPSK, 16-bit and 16QAM constellations are plotted on the unit circle shown in Figure 2-5. The minimum distance between the endpoints on the constellation is as follows

$(2-4)$

(2-5)

Where $\mathrm{L}$ is the number of levels projected on the horizontal and vertical axes of the signal points on the constellation.

Figure 2-5 16QAM and 16PSK constellation

When $\mathrm{M}=16$, we can calculate $0.47,0.39$, $>$, constellation, the greater the distance between the two signal points, noise interference in the case of fuzzy signal map, the need to separate the two possible signal points. By comparison, it is found that the anti - jamming ability of 16QAM system is better than 16PSK.

\subsection{QAM bit error rate performance}

The most prominent advantage of rectangular QAM signal constellation is that easy to produce PAM signal can be directly added to the two orthogonal carrier phase, in addition they are also easy to demodulate. For a rectangular signal constellation (where $\mathrm{k}$ is even) for $\mathrm{M}=$, the QAM signal constellation is equivalent to the two PAM signals on the quadrature carrier, and each of the two signals has a signal point. Since the signal on the phase quadrature component can be well separated by the coherence decision, it is easy to determine the 
bit error rate of the QAM by the bit error rate of the PAM. The probability of the correct judgment of the M-system QAM system is:

(2-6)

Where the bit error rate of the incoming PAM system has half of the average power of each quadrature signal of the equivalent QAM system. By appropriately adjusting the bit error rate of the M-system PAM system,

(2-7)

Where is the average signal-to-noise ratio for each symbol. So the M-bit QAM bit error rate is

(2-8)

When $\mathrm{k}$ is even, MQAM is accurate for $\mathrm{M}=$ case, and when $\mathrm{k}$ is an odd number, the equivalent PAM system cannot be found. If you use the best distance measure to determine the best decision, you can find any $\mathrm{k} 1$ bit error rate of the upper limit

Where is the average signal-to-noise ratio per bit.

\section{Chapter 3 Realization and Simulation of QAM Modulation Demodulation}

3.1 Simulink software

Simulink is application software that integrates with MATLAB software to simulate, simulate and analyze dynamic systems. It is based on the block diagram programming system simulation and simulation software, and to provide a basic system modeling module. Users only need to know the input and output of these modules and the function of the module, without having to look inside the module is how to achieve, through the basic module of the call, and then they can be connected to the required system model (in .mdl file To access), you can carry out simulation and analysis.

3.1.1 Simulink module

Simulink modules are divided into two categories, one is the basic module, and the other is the application-related modules. The basic module set consists of 16 modules, which are: common module, continuous time system module, discontinuous time system module, discrete time system module, logic and bit operation module, lookup table module, mathematical operation module, a model validation module, a system model tool module, a port and subsystem module, a signal attribute module, a signal routing module, a signal terminal module, a signal source module, a user defined module, other mathematical operations and discrete system modules.

Simulink's communication module, Communications Blockset provides a wealth of communication system simulation module, including almost all the communication system used in the simulation of the source, sink, operation and algorithms. As shown in Figure 3-1, users can use these modules to easily complete their own communication system simulation and analysis.

Figure 3-1 Communication system simulation module

\subsubsection{Simulink modeling and simulation}

The modeling steps are as follows:

(1) First, a system block diagram is designed, which describes the basic structure of the system, the flow of signals, the input and output of the subsystem signal, the interface between the subsystems, and the control required for system operation. This system block diagram helps Simulink build the system model.

(2) Start Simulink, in the Matlab command window (Command Window), enter simulink, the result is a desktop called Simulink Library Browser window, in this window lists the function of the classification of the various modules. You can also open the Simulink
Library Browser window with the shortcut buttons in the main frame of the Matlab.

(3) Click the New or Blank Model button under the File menu in the upper left corner of the Simulink module library window to open a blank model window named Untitled. Select the appropriate base module from Simulink's module library, point the mouse to the desired source module (sine wave, and press the left mouse button to drag it into the 'Untitled' window to generate a sine wave Source replicas.

(4) After selecting the module needed to build the system model, the module is connected according to the system block diagram. The connection method is as follows: the mouse arrow on the output port of the first module, which is the arrow will become a cross, click and continue to press the left mouse button, the mouse pointer to the second module input port, this is the mouse arrow into a double cross. Or use the mouse to select the source module, press the Ctrl key while clicking the target module.

After building a system model, the next thing is to run the model and get the simulation results. The complete process of running a simulation is divided into three steps: setting simulation parameters, starting simulation and simulation results analysis. Set the simulation parameters and select the solution, select the Simulation menu under the Parameters command, it will pop up a simulation parameters dialog box, which is mainly used Solver page WorkspaceI / O page Diagnostics page three pages to manage the simulation parameter settings. After setting the emulation parameters and selecting the solver, you can start the simulation and run it. Select the start option under the Simulink menu to start the simulation. If some parameters in the model are not defined, an error message will appear. If all settings are correct, start the simulation, system will issue a tweet in the end of simulation.

\subsection{QAM modulation module modeling and simulation}

The basic knowledge of QAM modulation and demodulation and Simulink is introduced, and the basic operation of communication simulation is introduced. In this paper, we used 16QAM as an example, using Simulink module to achieve QAM modulation, demodulation communication system modeling and simulation. By the second chapter MQAM modulation and demodulation principle drawn, 16QAM modulation and demodulation system block diagram shown in Figure 3-2:

Figure 3-2 16QAM modulation and demodulation

From Figure 3-2, we can know the principle of 16QAM modulation and demodulation, through the modulation and demodulation of the two modules to 16QAM system modeling and simulation, and the simulation results are analyzed, so the system is optimized, get better System model. The overall picture of the simulation system is shown in the appendix.

3.2.1 Source module

By analyzing the block diagram of the 16QAM modulation in Figure 3-1, the information carried by a symbol of 16QAM is 4 bits, which is twice the amount of information carried by the general baseband digital modulation (QPSK) symbol. And 16QAM modulation is modulated by two separate signals, a 16QAM symbol width is 2 times the base signal. Below is a brief introduction to the modules in the system simulation diagram.

This simulation uses the pseudo-random sequence generator in the signal source part. Since the system requires the baseband signal 
symbol rate of $19.2 \mathrm{kbps}$, the basic parameters of the sequence generator are set as follows:

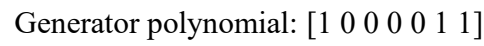

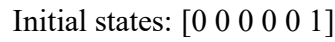

Output mask vector: 0

Sample time: $1 / 19200$

Output data type: double

3.2.2 Serial/parallel conversion module

As the system simulation of the total block diagram involves more modules, for the sake of beauty and easy to understand the string and transformed into a separate subsystem and embedded in the total system. The internal block diagram of the subsystem is shown in Figure 3-3:

Figure 3-3 serial and parallel conversion module

As can be seen from Figure 3-3, the subsystem has an input port and two output ports. The system first divides the input pseudorandom sequence into two and extracts one of them directly by the integer factor 2, and then carries out a unit delay, thus obtaining the odd-numbered symbols of the original random sequence; and then sampling to obtain the original sequence of even symbols.

Assume input In1: 001000001000011000101001

There are Out1: 010010010110

Out2: 000000001000001

The actual operation of the road signal shown in Figure 3-4, the figure from top to bottom are serial input, parallel output 1 and parallel output 2 waveform.

Figure 3-4 serial and parallel conversion of the signal map

It can be seen from the figure after the serial and parallel conversion, parallel output of each symbol transmission rate down to the original half, which is consistent with the theory. But each side of the output signal in front of a more 0-bit units, which is due to delay caused by the module, the performance of the back of the study will not be affected.

3.2.3 2/4 level conversion module

For the $2 / 4$ level conversion, in fact, the input signal of the four states $(00,01,10,11)$ after encoding for the corresponding 4-level signal. Here are the mapping relationships shown in Table 3-1:

Table 3-1 2/4 level mapping table

Pre-map data level /V
$00-3$
$01-1$
101
113

According to the above mapping, it is easy to find a mathematical relationship between them. Here the input signal is two binary signals, assuming that they are $\mathrm{ab}$, then in $\mathrm{a}=1$ to let it output a signal of amplitude 2 , when $\mathrm{a}=0$ output amplitude of -2 signal. Similarly, when $b=1$ is to let it output a signal of magnitude 1 , when $b$ $=0$ output amplitude of -1 signal. In this way you can get the following results:
When $\mathrm{ab}=00$ output: $\mathrm{y}=-2+-1=-3$;

$\mathrm{Ab}=01$ when $\mathrm{y}=-2+1=-1$;

$\mathrm{Ab}=10$ when $\mathrm{y}=2+-1=1$;

$\mathrm{Ab}=11 \mathrm{y}=2+1=3$;

From the above relationship: In the design of $2 / 4$ level conversion module, you need to first input the signal again and then serial and conversion, and then each signal to do a simple decision, and then add a module can be achieved 2 / 4 level conversion function. The specific modules are shown in Figure 3-5:

Figure 3-5 2/4 level conversion module

The signal diagram of each point in the above module is shown in Figure 3-6:

Figure 3-6 2/4 level conversion module points of the waveform

The first line in the figure for the input signal, the second three lines were serial and parallel conversion after the two lines of the signal, and finally for the output 4 level signal. Observe the waveform can be drawn:

Input: 000100100101101110

Parallel 1: 00001000111

Parallel 2: 01000110010

Output: $-3-1-31-1-11131$

Comparison of the waveform can be found in this module has been very good to achieve a $2 / 4$ level conversion, where the 4-level signal symbol transmission rate has been reduced to $\mathrm{Rb} / 4$.

3.2.4 The remaining modules

In addition to the above two subsystems, the modulation phase also includes the sine and cosine signal generator, the adder, the multiplier, the spectrum oscilloscope and the discrete time signal divergence diagram oscilloscope. Since the system requires a carrier frequency of $76.8 \mathrm{kHz}$, the parameters of the two-carrier signal generator are set as follows:

: Amlitude: 1: Amlitude: 1

Bias: 0 Bias: 0

Frequency $(\mathrm{rad} / \mathrm{sec}): 76800 * 2 *$ pi Frequency $(\mathrm{rad} / \mathrm{sec})$ : $76800 * 2 *$ pi

Phase (rad): pi / 2 Phase (rad): 0

Sample time: $1 / 768000$ Sample time: $1 / 768000$

For discrete time signal divergence diagram oscilloscope, here we do a sub-system shown in Figure 3-7:

Figure 3-7 Discrete-time signal divergence diagram oscilloscope

The above figure in the first two orthogonal signals and into a complex signal, the discrete sampling added to the signal divergence diagram oscilloscope, you can get the original signal constellation.

The above modules, subsystems according to the schematic diagram to connect, and the corresponding parameters of the module set, you can achieve its modulation function. The simulated output waveform shown in Figure 3-8 shows the constellation as shown in Figure 3-9:

Figure 3-8 16QAM modulation waveforms

In the above figure, the two-way parallel output of the two-way four-level signal, two four-line three lines were multiplied by the orthogonal carrier after the two-way signal. The fifth line is their sum signal, that is, the final modulation signal. 
Figure 3-9 16QAM constellations

\subsection{QAM demodulation module modeling and simulation}

The system previously obtained 16QAM modulation signal through the Gaussian white noise channel can be demodulated. 16QAM demodulation block diagram shown in Figure 3-2, the demodulator is the core of the $4 / 2$ level decision module and parallel conversion module.

The principle of the demodulator used in this paper is the coherent demodulation method, that is, the modulated signal is multiplied by the carrier and sent to the low-pass filter, which corresponds to the signal input and multiplied by the carrier through the LPF part, sent to the verdict. In this paper, the filters used in the simulation are selected Bessel low-pass filter. The parameters of the LPF are set as follows, and the output waveform is shown in 3-10.

Design method: Bessel

Filter type: Lowpass

Filter order: 8

Pass edge frequency $(\mathrm{rad} / \mathrm{s}): 15360 * 2 * \mathrm{pi}$

Figure 3-10

In the above figure, the three rows of the modulated wave are multiplied by the carrier, and the two or four lines are the waveforms after which they pass the low pass filter.

\subsubsection{4/2 level decision module}

Since the analog low-pass filter was used earlier, an analog 4level signal was obtained before the $4 / 2$ level decision. After wanting to get a 2-level digital signal, a series of samples, quantization and coding are required. The $4 / 2$ level decision subsystem is shown in Figure 3-11:

Figure 3-11 4/2 Level Translation Modules

In the above figure, the analog signal do a constant of 2 after the gain, let it pass a quantization encoder, and then through the discrete sampling will be a standard 4-level digital signal. And then the signal is divided into two respectively, after the quantization of the code obtained two binary signals, and finally by serial and conversion to get the final result. The parameters of the three quantization encoders here are as follows:

Quantization Encoder 2 Quantization partition: [-2.0 02.0$]$

Quantization codebook: [-3 -1 113$]$

Quantization Encoder 3 Quantization partition: [-2.0 02.0$]$

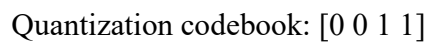

Quantization Encoder 4 Quantization partition: [-2.0 02.0$]$

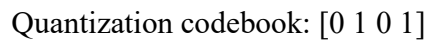

Assuming the above module input is $\mathrm{x}$, the output is $\mathrm{y}$, 、 then it completes the function is:

This two-way binary signal through the serial conversion, they completed the mapping shown in Table 3-2, but also the final realization of the $4 / 2$ level conversion.

Table 3-2 4/2 Level Mapping Table

Pre-map datalevel/V

$\begin{array}{lll}-3 & 00\end{array}$

$\begin{array}{lll}-1 & 01\end{array}$

110
$3 \quad 11$

The waveforms of the points in the above subsystems are shown in Figure 3-12.

Figure 3-12 4/2 level conversion in the point of the waveform

\subsubsection{Parallel/serial conversion module}

The parallel conversion module in the system consists of a pulse sequence generator and a selector. Where the pulse sequence generator is used to generate a full sequence with a duty cycle of 0.5 , and the selector is used to determine at which point which signal is to be output. Its parameters are set as follows:

Switch: Criteria for passing first input: $\mathrm{u} 2>=$ Threshold

Threshold: 0.5

Therefore, when the input pulse sequence is 1 , the selector outputs the first signal; when the input pulse sequence is 0 , the selector outputs the second signal. This simulation and after the string conversion will eventually achieve the 16QAM signal demodulation, the waveform shown in Figure 3-13:

Figure 3-13 16QAM demodulation output waveforms

In the above figure, the output of the $4 / 2$ decision device, the second line is the demodulated 16QAM final signal.

\subsubsection{Remaining modules}

In addition to the above modules, the demodulation phase is also used, including the eye diagram, the spread chart oscilloscope and error rate statistics and other information module. Figures 3-14 and 315 show the 16QAM signal constellation and the eye diagram before the $4 / 2$ decision when the channel SNR is $10 \mathrm{~dB}$.

Figure 3-14 16QAM signal plus noise after the constellation

Figure 3-15 Eye diagram of 16QAM signal

Research on QAM Anti - noise Performance

For square QAM, it can be seen as being composed of two mutually orthogonal and independent multi-levels ASK signals. Therefore, the use of multi-level error rate analysis method can be obtained M-band QAM bit error rate:

(3-1)

Where, for each symbol energy, for the noise unilateral power spectral density. Figure 3-16 shows the bit error rate curve for square QAM.

Figure 3-16 B-bit QAM BER curve

For 16QAM anti-noise performance simulation, as shown in Figure 16-16 16QAM error rate curve can be seen, when the large signal to noise ratio $(16 \mathrm{~dB})$ and the bit error rate for the level of personal computers to be calculated to such a time Long, so the simulation process will only calculate the accuracy to the level. As the actual simulation in many places will have a delay, so get the 16QAM demodulation signal to be a certain delay before it can be compared with the original random sequence. This delay is achieved through the error rate statistics module, the system overall block diagram Error Rate Calculation parameters set as follows:

Receive delay: 8

Computation delay: 0

Computation mode: Entire frame 
When the signal to noise ratio of more than $50 \mathrm{~dB}$, the system error rate has long been 0 , showing that this delay is no problem. By adjusting the signal-to-noise ratio SNR ( $\mathrm{Eb} / \mathrm{No}$ ) of the Gaussian white noise channel, the bit error rate shown in Figure 3-17 can be obtained as follows:

\section{Figure 3-17 16QAM bit error rate chart}

By comparing with Figure 3-16, it can be determined that the results obtained here are basically credible, that is, the simulation is successful. There are many factors in the simulation process that determine the anti-noise performance of the 16QAM modulation and demodulation system, such as the performance of the filter and the design of its passband, the position of the sampling decision, the setting of the decision threshold, and the time delay Or a small effect on its results.

Comparison of anti - noise performance between 16QAM and 2DPSK systems

This section designs a simulation model for 2DPSK modulation and demodulation systems to observe the anti-noise performance of the 16QAM signal and compare their bit error rates. At the same symbol rate and carrier rate, the 2DPSK modulation and demodulation system is designed as shown in Figure 3-18:

Figure 3-18 2DPSK modulation and demodulation system block diagram

2DPSK uses a differential coherent demodulation method and do not need to do carrier recovery. The demodulation part of the filter using a Bessel function low-pass filter, and the decision module shown in Figure 3-19:

\section{Figure 3-19 2DPSK decision module}

This adjusts the signal-to-noise ratio at the Gaussian white noise channel to obtain the bit error rate as shown in Figure 3-20:

Figure 3-20 16QAM and 2DPSK bit error rate curve

From the simulation process, in the same signal to noise ratio conditions, 16QAM additive white noise power is much larger than the 2DPSK additive white noise power, so 16QAM modulation and demodulation system generally work in the large signal to noise ratio environment. The bit error rate will be very small, but also the two systems under the same noise conditions, 16QAM anti-noise performance is quite superior.

\section{Chapter 4 Summary}

This paper focuses on the modulation and demodulation of modern digital modulation technology QAM, Simulink communication simulation software based on Matlab, 16QAM modulation and demodulation system as an example to design and simulation, to achieve the QAM system modulation and demodulation, and finally with the 2DPSK system were compared.

In this paper, the basic principles of MQAM modulation and demodulation system are deeply understood and analyzed, and Simulink simulation model is constructed with 16QAM modulation and demodulation principle. Master the Simulink software in the communication system design and simulation of the basic steps and methods. The design and simulation of the 16QAM modulation and demodulation system are realized, and the corresponding modulation and demodulation waveform is obtained. It is found that there is a certain delay in the waveform of the demodulated signal and the input signal, so the real-time performance of the system is not enough, but it does not affect the bit error rate detection, and the system can be antinoise performance.

Through the analysis and simulation of the anti - noise performance of 16QAM modulation and demodulation system, the error rate curve of 16QAM system is obtained. The curve trend is consistent with the theoretical curve. Comparing the anti-noise performance of 16QAM modulation and demodulation system with 2DPSK system, the error rate curve of both 16QAM systems is compared. It is found that the anti-noise performance of 16QAM is not as good as 2DPSK, that is, when the signal-to-noise ratio is constant, $\mathrm{M}$ The greater the bit error rate $\mathrm{Pe}$ is also greater. From the simulation results, in the same signal to noise ratio conditions, 16QAM additive white noise power is much larger than the 2DPSK additive white noise power, so 16QAM modulation and demodulation system generally work in the large signal to noise ratio environment, the bit error rate will be very small. That is, the two systems under the same noise conditions, 16QAM anti-noise performance is quite superior.

\section{REFERENCES}

[1] Fan Changxin, Cao Lina. Communication principle [M]. Beijing: National Defense Industry Press, 2006.9

[2] Zhang Hui, Cao Lina. Modern communication principles and technology [M]. Xi'an: Xi'an University of Electronic Science and Technology Press, 2008.7

[3] Cao Zhigang. Modern communication principle [M]. Beijing: Tsinghua University Press, 2007.8

[4] Da Xinyu. Communication Principles Tutorial [M]. Beijing: Posts and Telecommunications University Press, 2005.1

[5] Li He Bing. Simulink communication simulation tutorial [M]. Beijing: National Defense Industry Press, 2006.5

[6] Li Yan .MATLAB Communication Simulation Development Manual [M]. Beijing: National Defense Industry Press, 2005.1

[7] Li Jianxin. Modern communication system analysis and simulation -MATLAB communication toolbox [M]. Xi'an: Xi'an University of Electronic Science and Technology Press, 2000.8

[8] Jia Qiuling.MTLAB7. X / Simulink / Stateflow System Simulation, Analysis and Design $[\mathrm{M}]$. Xi'an: Northwestern Polytechnical University Press, 2006.6

[9] Sun Yi, Wu Lei. Simulink communication simulation development manual [M]. Beijing: National Defense Industry Press, 2004.10

[10] Shao Ji.MATLAB / Simulink communication system modeling and simulation examples fine [M]. Beijing: Electronic Industry Press, 2009.7

[11] Liu Min. MATLAB communication simulation and application [M]. Beijing: National Defense Industry Press, 2001.1 